\title{
Fixação cirúrgica de fratura oblíqua de mandíbula utilizando lag screw: relato de caso
}

Surgical fixation of mandible oblique fracture using lag screw: case report

Fijacíón cirúrgica de fractura oblicua de mandíbula utilizando lag screw: relato de caso

Maísa de Oliveira AGUILLERA ${ }^{1}$

Gustavo Silva PELISSARO ${ }^{2}$

José Carlos Garcia de MENDONÇA ${ }^{3}$

Janayna Gomes PAIVA-OLIVEIRA ${ }^{2}$

Alana Oswaldina Gavioli Meira dos SANTOS ${ }^{4}$

Ellen Cristina GAETTI-JARDIM ${ }^{5}$

${ }^{I}$ Residente em Cirurgia e Traumatologia Bucomaxilofacial, Hospital Universitário Maria Aparecida Pedrossian, Universidade Federal de Mato Grosso do Sul (UFMS)

${ }^{2}$ Preceptor da Residência em Cirurgia e Traumatologia Bucomaxilofacial do Hospital Universitário Maria Aparecida Pedrossian. Universidade Federal de Mato Grosso do Sul (UFMS)

${ }^{3}$ Coordenador da Residência em Cirurgia e Traumatologia Bucomaxilofacial do Hospital Universitário Maria Aparecida Pedrossian, Universidade Federal de Mato Grosso do Sul (UFMS)

${ }^{4}$ Mestranda. Programa de Pós-graduação Saúde e Desenvolvimento da Região Centro-Oeste da Universidade Federal de Mato Grosso do Sul (UFMS)

${ }^{5}$ Tutora da Residência em Cirurgia e Traumatologia Bucomaxilofacial do Hospital Universitário Maria Aparecida Pedrossian, Universidade Federal de Mato Grosso do Sul (UFMS)

\section{Resumo}

A mandíbula se configura como um dos ossos mais acometidos por trauma no esqueleto maxilofacial em virtude de sua projeção anteriorizada. Sua localização está associada a direção e força do impacto, ocorrendo mais frequentemente na região de ângulo mandibular seguidas de sínfise e corpo. O tratamento de qualquer tipo de fratura consiste na redução e fixação dos fragmentos ósseos, o mais precoce possível, assim que as condições gerais do paciente permita. Este trabalho tem como objetivo descrever uma das técnicas de fixação interna rígida mandibular, utilizando parafusos na técnica lag screw. Relato de caso: Paciente, 37 anos, gênero feminino, compareceu ao pronto atendimento do Hospital Maria Aparecida Pedrossian com histórico de agressão física cursando com dificuldades de mastigação, apresentando ao exame tomográfico fratura de corpo mandibular com moderado deslocamento e em posição oblíqua. Diante do exame clinico e tomográfico, optou-se como conduta terapêutica o tratamento cirúrgico sob anestesia geral por meio da técnica lag screw que se mostrou uma técnica com resultado satisfatório até o momento e menos oneroso para o sistema publico de saúde.

Descritores: Mandíbula; Fixação de Fratura; Cirurgia Bucal.

\section{Abstract}

The mandible is configured as one of the bones most affected by trauma in the maxillofacial skeleton due to its anterior projection. Its location is associated with the direction and force of the impact, occurring in the mandibular angle region followed by symphysis and body more often. The treatment of any type of fracture consists of the reduction and fixation of bone fragments, as early as possible, as soon as the patient's general conditions permit. This article aims to describe one of the internal rigid mandibular fixation techniques, using screws in the lag screw technique. Case report: A 37-year-old female patient attended the emergency room at the Hospital Maria Aparecida Pedrossian with a history of physical aggression, with chewing difficulties, presenting a tomographic examination of the mandibular body with moderate displacement and in an oblique position. In view of the clinical and tomographic examination, surgical treatment under general anesthesia was chosen as a therapeutic approach using the lag screw technique, which proved to be a technique with satisfactory results so far and less costly for the public health system.

Descriptors: Mandible; Fracture Fixation; Surgery Oral.

\section{Resumen}

La mandíbula está configurada como uno de los huesos más afectados por un traumatismo del esqueleto maxilofacial debido a su proyección anterior. Su ubicación está asociada con la dirección y la fuerza del impacto, ocurriendo con más frecuencia en la región del ángulo mandibular seguido de la sínfisis y el cuerpo. El tratamiento de cualquier tipo de fractura consiste en la reducción y fijación de los fragmentos óseos, lo antes posible, tan pronto como lo permitan las condiciones generales del paciente. Este trabajo tiene como objetivo describir una de las técnicas de fijación mandibular rígida interna, utilizando tornillos en la técnica lag screw. Relato de caso: Paciente de 37 años acudió a la sala de urgencias del Hospital María Aparecida Pedrossian con historia de agresión física, con dificultades para masticar, presentando al examen tomográfico fractura del cuerpo mandibular de manera oblicua y moderada. En vista del examen clínico y tomográfico, la conducta terapéutica elegida fue el tratamiento quirúrgico bajo anestesia utilizando la técnica lag screw, que ha demostrado ser una técnica con resultados satisfactorios hasta ahora y menos costosa para el sistema de salud público.

Descriptores: Mandíbula; Fijación de Fractura; Cirurgía Bucal.

\section{INTRODUÇÃO}

A mandíbula é a segunda parte do esqueleto maxilofacial com maior acometimento de fratura, devido a sua projeção anteriorizada, perdendo somente para a fratura dos ossos nasais ${ }^{1}$. As principais causas de fraturas faciais incluem os acidentes automobilísticos e as agressões. Outras causas são as quedas, os acidentes esportivos e os acidentes de trabalho, sendo o sexo masculino mais acometido e as fraturas de mandíbula como a segunda mais incidente com $42.5 \%$ dos casos analisados ${ }^{2}$.

Dependendo do tipo de lesão, da direção e da força do impacto, as fraturas mandibulares podem ocorrem em diversas localizações sendo as fraturas de ângulo com maior prevalência, seguidas da sínfise e corpo mandibular ${ }^{3,4}$. O tratamento destas fraturas consiste na redução e fixação dos fragmentos ósseos, o mais precoce possível, assim que as condições gerais do paciente permitirem.

Muitas técnicas são descritas na literatura para realização de osteossintese de fraturas mandibulares. Placas de reconstrução, parafusos do tipo lag screw, e sistemas de duas placas, foram todos desenvolvidos com a intenção de minimizar as lacunas interfragmentárias para alcançar a cura óssea primária ${ }^{5}$.

A osteossintese utilizando parafusos foi descrita pela primeira vez em 1970, por Brons e Boering ${ }^{6}$ demonstrando como técnica cirúrgica a fixação de dois fragmentos ósseos com parafusos perfurando ambas as corticais, proporcionando assim uma estabilidade aceitável da fratura ${ }^{7}$. A mesma 
consiste na compressão de fraturas que pode ser realizada utilizando parafusos específicos para a técnica ou parafusos ósseos longos, aplicada em fraturas que correm obliquamente ${ }^{8}$. A fixação deve ser capaz de resistir as forças de deslocamento que agem contra a mandíbula, levando em consideração a ação muscular? .

Este trabalho tem como objetivo descrever uma das técnicas de fixação interna rígida mandibular, utilizando parafusos na técnica lag screw, demonstrando a facilidade, viabilidade $\mathrm{e}$ resultado satisfatório da técnica, assim como o menor custo para o sistema único de saúde pública.

\section{CASO CLÍNICO}

Paciente, 37 anos, gênero feminino, compareceu ao pronto atendimento do Hospital Maria Aparecida Pedrossian com histórico de agressão física cursando com dificuldades de mastigação. Ao exame extraoral paciente apresentou edema em região submandibular do lado esquerdo e dificuldades de abertura bucal, degrau ósseo a palpação em região de corpo mandibular esquerdo e algia local. Ao exame clínico intrabucal, apresentou ausências dentárias, abaulamento presente em parede lingual de mandíbula esquerda com presença de ulceração ipsilateral. Ao exame tomográfico apresentou fratura obliqua em região de corpo mandibular esquerdo com grande disjunção dos cotos ósseos (Figuras 1 e 2).
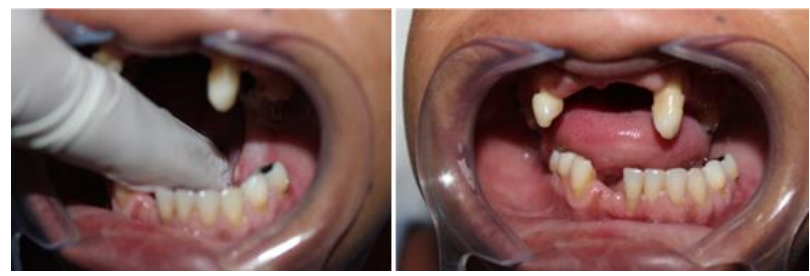

Figura 1: Aspecto intraoral inicial. Nota-se ulceração em tábua lingual e desvio mandibular para o lado esquerdo.

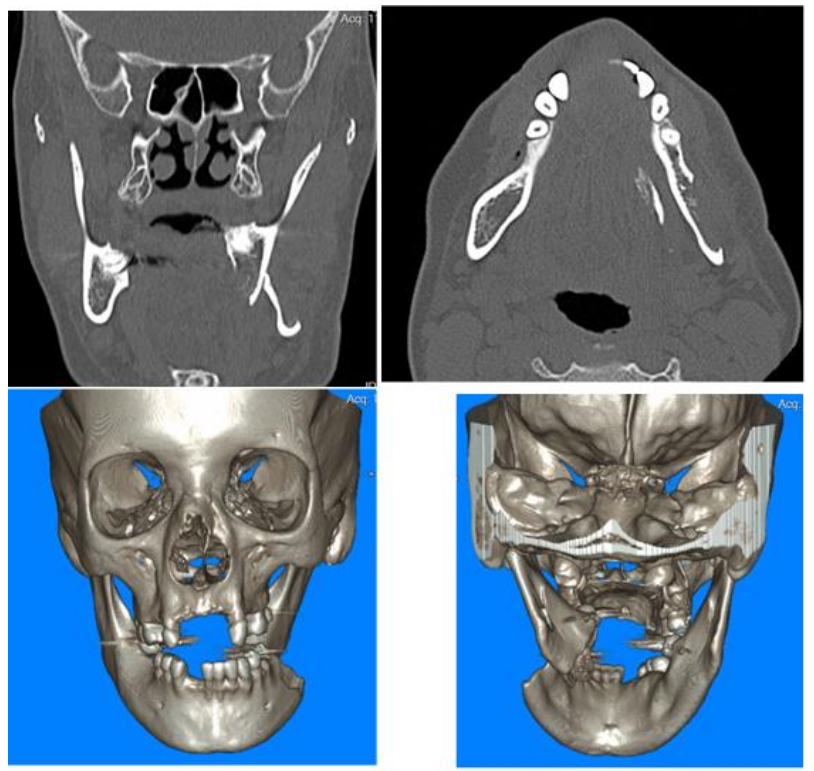

Figura 2: Tomografia pré-operatória, evidenciando fratura do corpo mandibular com deslocamento medial do coto proximal.
Diante do exame clinico e tomográfico, optou-se como conduta terapêutica o tratamento cirúrgico sob anestesia geral por meio da técnica lag screw, no terceiro dia da internação hospitalar, após remissão do edema. Sob anestesia geral e intubação nasotraqueal procedeu-se a assepsia intra e extraoral com clorexidina $0.12 \%$ e Clorexidina alcoólica $0.5 \%$, respectivamente e aposição de campos estéreis. Foram realizadas infiltrações com lidocaína a $2 \%$ com epinefrina 1:100.000 em região vestibular de mandíbula esquerda. Os segmentos fraturados foram abordados por acesso intraoral em região vestibular de mandíbula e realizado redução cirúrgica com auxílio de pinça Kocher, e os fragmentos fixados com três parafusos longos de $15 \mathrm{~mm}$ do sistema 2.0 pela técnica lag screw por meio de trocater. Em virtude da falta de uma oclusão estável e a complexidade de redução da fratura, não foi realizado o bloqueio intermaxilar trans-operatório. Em seguida foi realizado a sutura mucoperiosteal com monocryl 4-0 violeta (Figura 3). No pós-operatório imediato, paciente apresentou edema compatível com o procedimento cirúrgico, ausência de sangramento intraoral, sutura de acesso em posição, higiene oral regular, parestesia em lábio inferior esquerdo e ausência de queixas álgicas. Ao exame tomográfico de controle pós-operatório apresentou boa redução e fixação dos cotos ósseos, recebendo assim alta hospitalar no primeiro dia pós-operatório (Figura 4).
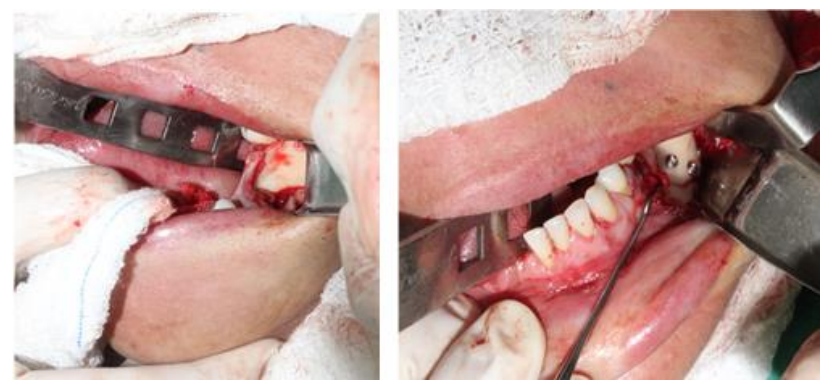

Figura 3: Exposição da fratura. Fixação pela técnica de lag screw.
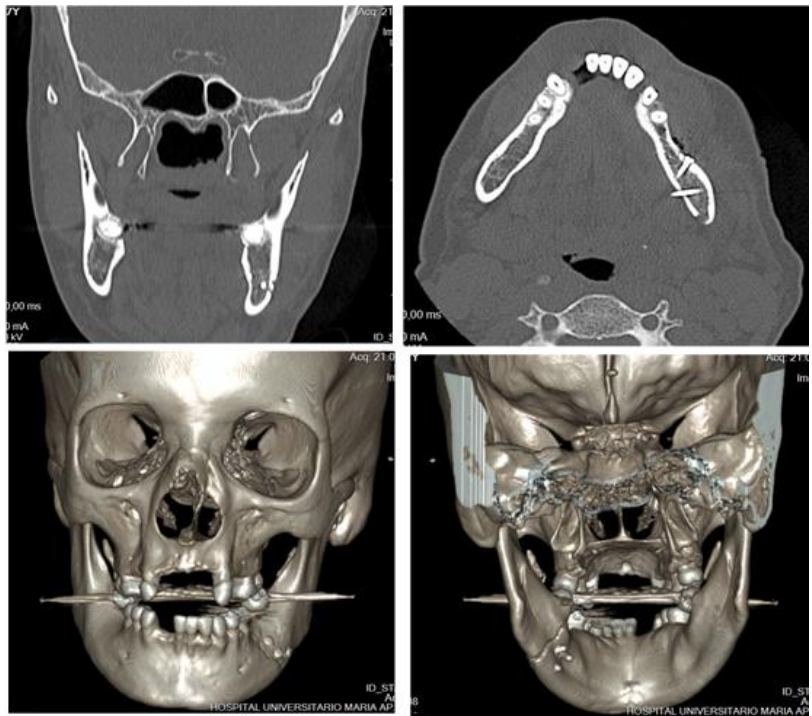

Figura 4: Tomografia pós-operatória apresentando adequada redução fixação da fratura. 
No sexto dia pós-operatório paciente retornou para acompanhamento clinico relatando oclusão dentária preservada, ausência de algia, neuropraxia do nervo marginal da mandíbula do nervo facial do lado esquerdo, apresentando melhora de parestesia de lábio inferior esquerdo. Clinicamente apresentou ausência de equimose ou edema em face, intraoral, sutura de acesso cirúrgico bem posicionada, com ausência de sinais flogísticos e oclusão satisfatória. Após dois meses de acompanhamento ambulatorial, a paciente ainda refere parestesia em região de lábio inferior esquerdo, oclusão dentaria preservada, sem limitação de abertura bucal e intraoral apresentou boa cicatrização de acesso cirúrgico sem sinais de infecção local.

DISCUSSÃO

Para a realização de redução e fixação de uma fratura com grande deslocamento, visando a estabilidade dos cotos ósseos, faz-se necessário a utilização de anestesia geral para o conforto do paciente.

A fim de promover uma cirurgia em menor tempo operatório, com completa visão do campo, a literatura preconiza que, em procedimentos na região anterior da mandíbula, o acesso seja realizado através de uma incisão intraoral. Para tal, a incisão é realizada na região vestibular da mandibula desde a prega gengivolabial poucos milímetros abaixo da gengiva inserida e até o periósteo onde preserva a mucosa facilitando o reparo estendo o suficiente para visualização completa da fatura ${ }^{10}$. Assim sendo, o caso apresentado corrobora com os artigos com a confecção do acesso intrabucal com resultado satisfatório.

$\mathrm{O}$ acesso intraoral permite a visualização direta da oclusão durante a redução e fixação da fratura, diminui o tempo cirúrgico e a incidência de lesão de estruturas nervosas, como o ramo mandibular do nervo facial, além de proporcionar uma cicatriz oculta ${ }^{11}$. Contudo, o nervo alveolar inferior pode ser danificado durante a redução cirurgica e reparo de fratura mandibula um dos pontos analisados para a escolha do acesso do caso em questão. $\mathrm{O}$ acesso extrabucal é consagradamente realizado a fim de visualização mais facilitada e direta para abordagem ao local da fratura. Aqui, o acesso intrabucal nao aumentou o tempo pósoperatório e demonstrou resultados esperados de redução e fixação da fratura ${ }^{10}$.

Outro fato relevante, apesar do grande deslocamento da fratura mas com localização corpo anterior de mandíbula, foi optado pelo acesso intraoral com auxílio de trocarte para fixação dos parafusos pela técnica lag screw, o que permitiu visualização no trans-operatório de uma oclusão clinica pois não foi possível a realização de bloqueio intermaxilar pela instabilidade de oclusão dentária.
Segundo Ramalho et al. ${ }^{12}$, as fraturas de corpo mandibular biseladas são difíceis de redução e fixação, o que se mostrou evidente no ato cirúrgico, a pesar da boa exposição da fratura sendo necessário o auxílio de pinças de redução para evitar distanciamento dos cotos ósseos.

A técnica lag screw baseada na fixação dos fragmentos ósseos com parafusos bicorticais, mostrando-se mais efetivo em fraturas bizeladas promovendo rápida fixação sem diminuir a rigidez da redução ${ }^{13}$.

Esta técnica permite um menor tempo cirúrgico por sua simplicidade, podendo esta ser realizada mediante acesso intraoral transcorrendo em um pós-operatório com menor morbidade ${ }^{7}$ sendo este um dos da conduta cirúrgica adotada.

CONCLUSÃO

Em vista da variedade de técnicas cirúrgicas e tratamentos das fraturas mandibulares, cabe a cada cirurgião bucomaxilofacial a escolha de acordo com a experiência profissional. Deste modo, a instituição da técnica lag screw, mostrou-se uma técnica com resultado satisfatório até o momento e menos oneroso para o sistema público de saúde.

\section{REFERÊNCIAS}

1. Flandes MP, Dias LBGM, Paulesini Junior W. Fratura de mandíbula - relato de caso. Rev Odontol Univ Cid São Paulo. 2019; 31(2):205-12.

2. Zamboni RA, Wagner JCB, Volkweis MR, Gerhardt EL, Buchmann EM, Bavaresco CS. Levantamento epidemiológico das fraturas de face do Serviço de Cirurgia e Traumatologia Bucomaxilofacial da Santa Casa de Misericórdia de Porto Alegre - RS. Rev Col Bras Cir. 2017; 44(5):491-97.

3. Dantas BPSS, Fialho PV, Fernandes ACF, Silva DT, Queiroz CS. Fratura complexa de mandíbula: relato de caso. Rev Odontol Araçatuba. 2017; 38(3):43-8.

4. Hupp JR, Ellis III E, Tucker MR. Cirurgia Oral e Maxilofacial. 5.ed. Rio de Janeiro: Elsevier; 2009.

5. Wang R, Liu Y, Wang JH, Baur DA. Effect of interfragmentary gap on the mechanical behavior of mandibular angle fracture with three fixation designs: A finite element analysis. J Plast Reconstr Aesthet Surg. 2017;70(3):360-69.

6. Brons R, Boering G. Fractures of the mandibular body treated by stable internal fixation: a preliminary report. J Oral Surg. 1970;28(6):407-15.

7. Campolongo GD, Barros TP, Amantéa DV. Fixação das fraturas mandibulares, utilizando a técnica lag screw associada à sedação: relado de caso. Rev Cir Traumatol Buco-Maxilo-Fac. 2005; 5(1):39-44.

8. Fonseca RJ, Walker RV, Barber HD, Powers MP, Frost DE. Trauma bucomaxilofacial. 4.ed. Rio de Janeiro: Elsevier; 2015. 
9. Miloro M, Ghali GE, Larsen PE, Waite PD. Princípios de cirurgia bucomaxilofacial de Peterson. 3.ed. São Paulo: Santos; 2016.

10. El-Anwar MW, Sweed AH, Abdulmonaem G. Mental Foramen Relation to Mandibular Fracture. J Craniofac Surg. 2016;27(8):e743-e745.

11. Mendonça JCG, Gaetti Jardim EG, Manrique GR, Freitas GP. Acesso cirúrgico para tratamento de fraturas mandibulares: Revisão de literatura. Arch Heath Invest. 2013;2(2):19-23.

12. Ramalho RA, Araújo FAC, Santos FSM, Caubi AF, Sobreira T. Tratamento de fratura de mandibula: miniplacas e parafusos x lag screws relato de caso. Rev Cir Traumatol Buco-MaxiloFac. 2011;11(1):9-12.

13. Pereira ICS, Galdino AS, Palitó APPG, Santos TL, Barros F, Sobreira T. Redução cirúrgica de fratura mandibular oblíqua: parafusos transcorticais x placas de titânio - relatos de caso. Rev Cir Traumatol Buco-Maxilo-Fac. 2011;11(3):69-76.

\section{CONFLITO DE INTERESSES}

Os autores declaram não haver conflitos de interesse.

\section{AUTOR PARA CORRESPONDÊNCIA}

\section{Maísa de Oliveira Aguillera}

Programa de Residência em Cirurgia e Traumatologia Bucomaxilofacial

Hospital Universitário Maria Aparecida Pedrossian, UFMS/EBSERH

(67) 3345-3140

E-mail: ctbmfhumap@gmail.com
Submetido em 16/04/2020

Aceito em 08/09/2020 